\title{
Effects of basis set and electron correlation on the calculated properties of the ammonia dimer
}

\author{
Z. Latajka ${ }^{a)}$ and Steve Scheiner),c) \\ Department of Chemistry and Biochemistry, Southern Illinois University, Carbondale, Illinois 62901
}

(Received 8 March 1984; accepted 21 March 1984)

\begin{abstract}
$A b$ initio calculations are carried out for $\left(\mathrm{NH}_{3}\right)_{2}$ with a $6-31 \mathrm{G}^{* *}(1 p, 2 d)$ basis set containing diffuse polarization functions. Electron correlation is included via second-order Mфller-Plesset perturbation theory (MP2). At the SCF level, the equilibrium $R$ (NN) distance is $3.54 \AA$ and the interaction energy is $-2.35 \mathrm{kcal} / \mathrm{mol}$. Inclusion of correlation enhances the attraction substantially, increasing the energy to $-4.05 \mathrm{kcal} / \mathrm{mol}$ and reducing the intermolecular separation by $0.20 \AA$. Comparison with previous results at the SCF level demonstrates a variety of errors including exaggerated dipole moments, underestimation of polarization energy, and sizable superposition errors with these smaller basis sets.
\end{abstract}

The ammonia dimer represents perhaps the simplest and most straightforward prototype of the $\mathrm{NH}-\mathrm{N} \mathrm{H}$ bond. The molecular interaction is important also in connection with elucidation of effective pair potentials suitable for modeling the solid and liquid states of ammonia. Whereas the water dimer, which is the oxygen analog of $\left(\mathrm{NH}_{3}\right)_{2}$, has been the subject of a great deal of theoretical attention including calculations of very high degree of accuracy, ${ }^{1-6}$ the dimer of ammonia has been largely neglected. Most $a b$ initio studies have been limited to the SCF level with basis sets of only moderate size. ${ }^{7-12}$ In only one study was electron correlation considered but this work neglected effects of correlation upon the geometry of the complex. ${ }^{11}$ Moreover, the basis set used was $6-31 G^{* *}$, which is well known to exaggerate the dipole moment and in addition, as we have recently shown, is not sufficiently flexible for an adequate treatment of correlation. ${ }^{13}$ In the present communication, we report calculations of the ammonia dimer that explicitly include the effects of correlation upon the intermolecular geometry as well as the $\mathrm{H}$-bond energy; a basis set is used which leads to a dipole moment in good agreement with experiment and which contains two sets of polarization functions for proper treatment of electron correlation.

\section{DETAILS OF CALCULATIONS}

Geometries considered were those belonging to the $C_{s}$ point group and which contain a linear arrangement of the $\mathbf{N}-\mathbf{H} \rightarrow \mathbf{N} \mathbf{H}$ bond. The $\mathrm{H}$-bond axis was taken as identical to the local $C_{3}$ rotation axis of the proton-acceptor $\mathrm{NH}_{3}$ molecule. The two hydrogens of the proton-donating subunit were staggered with respect to the hydrogens of the other molecule. This type of intermolecular arrangement has been demonstrated previously to be most stable for the ammonia dimer. ${ }^{7,9,10}$ The internal structures of each $\mathrm{NH}_{3}$ subunit were held fixed in the experimentally determined geometry ${ }^{14}$ of the monomer: $r(\mathrm{NH})=1.0124 \AA ; \theta(\mathrm{HNH})=1.06 .68^{\circ}$.

\footnotetext{
a) On leave from the Institute of Chemistry, University of Wroclaw, Wroclaw, 50-383 Poland.

b) Author to whom reprint requests should be addressed.

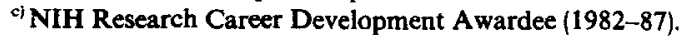

We expect little error to be introduced by the latter assumption of fixed internal geometries due to the weak nature of the $\mathrm{H}$ bond.

$A b$ initio calculations were carried out using the GAUSSIAN-80 package of computer codes. ${ }^{15}$ Electron correlation was considered via Møller-Plesset perturbation theory to second order (MP2), ${ }^{16,17}$ keeping the cores of the nitrogen atoms frozen. As a choice of basis set, we modified 6-31G** by adding a second and diffuse set of $d$-functions $(\xi=0.25)$ to the nitrogens. ${ }^{18} \mathrm{~A}$ single set of diffuse $p$-functions with orbital exponent 0.15 was used for hydrogen. This basis set, denoted as 6-31G** $(1 p, 2 d)$, has been shown to yield excellent estimates of the electric multipole moments of $\mathrm{NH}_{3}$, to minimize basis set superposition errors, and to provide a good framework for electron correlation. ${ }^{13}$

\section{RESULTS}

The intermolecular distance was optimized at both the SCF and MP2 levels. These results are presented in Table I along with data from previous calculations with various basis sets ranging in size from STO-3G ${ }^{8,9}$ on the far left to the [541/31] basis set ${ }^{10}$ of Hinchliffe et al. There is a clear trend toward longer intermolecular separation as the basis set is improved; i.e., from left to right in Table I. For example, the $R$ (NN) distance is $3.1 \AA$ with the minimal basis set, increases to $3.3 \AA$ with the split-valence $4-31 \mathrm{G}$ set, and lengthens further to $3.4 \AA$ when polarization functions are added. Augmenting the basis with a second set of $d$-functions produces an additional increase to $3.54 \AA$. However, inclusion of electron correlation leads to a substantial reduction in the intermolecular distance by $0.2 \AA$. Indeed, it should be noted that the MP2 value of $R(N N)$ is quite similar to various experimental estimates in the condensed phases ${ }^{19,21}$; there is no currently available value for the dimer in the gas phase.

The trends in the interaction energy $\Delta E$ in the second row of Table I are not quite as simple as for $R$. The general pattern involves an overall reduction in $\Delta E$ as the basis set is enlarged. The unpolarized STO-3G and 4-31G basis set results are clustered around $-4 \mathrm{kcal} / \mathrm{mol}$ with $4-31 \mathrm{G}$ being somewhat higher. The values for the singly polarized $6-31 G^{*}$ and 6-31G** basis sets are around -3 . [541/31], like 6$31 G^{* *}(1 p, 2 d)$, contains two sets of $d$-functions although they 
TABLE I. Properties of $\mathrm{NH}_{3}$ dimer.

\begin{tabular}{|c|c|c|c|c|c|c|c|c|c|}
\hline & STO-3G & STO-3G & $4-31 G$ & $6-31 G^{*}$ & $6-31 G^{* *}$ & {$[541 / 31]$} & $6-31 \mathrm{G} * *(1 p, 2 d)$ & $\mathrm{MP}^{\mathrm{a}}$ & Experiment \\
\hline$R(\mathrm{NN}), \AA$ & $3.067^{b}$ & $3.08^{c}$ & $3.31^{\mathrm{c}}$ & $3.44^{\mathrm{c}}$ & $3.44^{\mathrm{d}}$ & $3.44^{e}$ & 3.540 & 3.336 & $\begin{array}{l}3.35 \text { (crystal) }^{f} \\
3.37 \text { (liquid) }\end{array}$ \\
\hline$\Delta E, \mathrm{kcal} / \mathrm{mol}$ & $-3.74^{b}$ & $-3.8^{\mathrm{c}}$ & $-4.1^{\mathrm{c}}$ & $-2.9^{c}$ & $-3.11^{h}$ & $-2.4^{c}$ & -2.35 & -4.05 & $-(4.4-4.6)^{i}$ \\
\hline BSSE, kcal/mol & -3.08 & -3.05 & -1.97 & -0.53 & -0.55 & -1.04 & -0.68 & $\ldots$ & $\ldots$ \\
\hline$\Delta E$-BSSE, $\mathrm{kcal} / \mathrm{mol}$ & -0.66 & -0.8 & -2.1 & -2.4 & -2.56 & -1.4 & -1.67 & $\cdots$ & $\ldots$ \\
\hline$\mu\left(\mathbf{N H}_{3}\right), \mathrm{D}$ & 1.79 & 1.66 & 2.28 & 1.93 & 1.87 & 1.84 & 1.50 & $\cdots$ & $1.47^{j}$ \\
\hline
\end{tabular}

With $6-31 \mathrm{G}^{* *}(1 p, 2 d)$ basis set.

${ }^{b}$ From Ref. 9.

${ }^{c}$ From Ref. 8.

d Taken from 6-31G* optimization.

e From Ref. 10.

${ }^{\mathrm{f}}$ References 19 and 20.

${ }^{B}$ Reference 21.

"From Ref. 11.

${ }^{\mathrm{i}} \Delta H_{298}^{\circ}$ (see the text) from Refs. 22-24.

${ }^{\text {i }}$ Reference 25.

are contracted into a single shell in the former case. ${ }^{10}$ Values of $\Delta E$ with these basis sets are approximately $-2.4 \mathrm{kcal} /$ mol. When correlation is included, a sizable increase in the binding energy is observed; the MP2 value is $72 \%$ higher than the SCF energy. The experimental energies listed in the last column are somewhat misleading in that they refer to $\Delta H^{\circ}$ at $298 \mathrm{~K}$ while the theoretical values correspond to the electronic energy at $0 \mathrm{~K}$. More will be said of this comparison below.

The high interaction energies and small values of $R$ computed with the small basis sets are suggestive of sizable basis set superposition errors (BSSE). The magnitude of the BSSE was computed by the Boys and Bernardi counterpoise scheme ${ }^{26}$ and is presented in the third row of Table I for each basis set. The unpolarized basis sets are subject to errors of 2 or $3 \mathrm{kcal} / \mathrm{mol}$ which may certainly not be neglected in view of the small interaction energies in the ammonia dimer. The superposition errors for the other basis sets are generally equal to about $0.5 \mathrm{kcal} / \mathrm{mol}$ with a somewhat higher value for [541/31]. The next row of Table I contains the interaction energy after being corrected by subtraction of the BSSE. The patterns observed are quite different than for the uncorrected $\Delta E$. The minimal $\mathrm{STO}-3 \mathrm{G}$ basis set leads to very small $H$-bond energies with progressively higher values obtained with $4-31 G, 6-31 G^{*}$, and $6-31 G^{* *}$. Subsequent improvement of the basis set is associated with decrease in the corrected energies.

It is possible to gain some insights into the reasons underlying the above trends by partitioning the interaction energy into its various components. The electrostatic contribution is expected to play a large role and will be dominated by the dipole-dipole term. We have therefore included in the last row of Table I the dipole moment of $\mathrm{NH}_{3}$ computed with each basis set. The STO-3G dipole moments are slight overestimates of the experimental value of $1.47 \mathrm{D}$ and hence the electrostatic component is expected to be treated reasonably well. However, this basis set is subject to an extremely large BSSE which dominates the computed value. The very small corrected interaction energies are likely due in large part to underestimates of the polarization energy resulting from the inflexibility of the minimal basis set. The polarization component is probably treated somewhat better with 4-31G; however, this basis set is plagued by severe exaggeration of the dipole moment (and hence electrostatic attraction) and by a substantial BSSE. The latter error is much reduced with the polarized $6-31 G^{*}$ and $6-31 G^{* *}$ basis sets but these also suffer from overestimates of the dipole moment, albeit not as severely as 4-31G. The fact that the polarized $6-31 \mathrm{G}^{*}$ and 6 $31 \mathrm{G}^{* *}$ basis sets lead to a greater corrected interaction energy than does 4-31G probably reflects the better treatment of the polarization attraction with the more flexible basis sets. Addition of diffuse polarization functions in 6-31G**(1p,2d) produces a marked drop in the interaction energy due principally to a lower and more realistic value of the $\mathbf{N H}_{3}$ dipole moment (and quadrupole moments ${ }^{13}$ as well). A second factor may be a slightly increased exchange repulsion resulting from overlap between the diffuse orbitals of the two subsystems. The results with the [541/31] basis sets are fairly anomalous for a number of reasons. First, the BSSE is surprisingly large for a basis set of this size. Thus, even though the uncorrected interaction energies of this basis set and 6$31 \mathrm{G}^{* *}(1 p, 2 d)$ are nearly identical, the corrected value of the former is somewhat smaller in magnitude. Indeed, this small value is particularly surprising in view of the [541/31] overestimate of the dipole moment of $\mathrm{NH}_{3}$. It would appear that the contracted nature of the $d$-orbital used (primitive exponents of 1.97 and 0.58 ) does not allow an adequate description of the attractive polarization energy. The size and flexibility of the $6-31 \mathrm{G}^{* *}(1 p, 2 d)$ basis set therefore offers the best treatment of the various components of the interaction and moreover leads to a small superposition error.

As mentioned above, electron correlation plays a major role in stabilization of the $\mathrm{NH}_{3}$ dimer. The second-order correction amounts to $-1.70 \mathrm{kcal} / \mathrm{mol}$ which is $42 \%$ of the total interaction energy of $-4.05 \mathrm{kcal} / \mathrm{mol}$. The only previous calculation of the contribution of correlation to the stability of this system is a MP treatment with the 6-31G** basis set by Pople. ${ }^{11} \mathrm{He}$ found an MP2 correction of -1.2 $\mathrm{kcal} / \mathrm{mol}$, a slightly smaller value which is not surprising in light of the lesser flexibility of the singly polarized basis set.In addition, this correction was calculated at an intermolecular distance optimized at the SCF level (with a different basis set) and does not reflect the full magnitude of correlation effect. Pople's results are useful, however, in that they provide an indication of the magnitude of higher order corrections. Third-order contributions were found to be repulsive by $0.24 \mathrm{kcal} / \mathrm{mol}$ and partial fourth order (MP4SDQ) by 0.05 . We conclude that our second-order correction of $-1.7 \mathrm{kcal} / \mathrm{mol}$ is probably somewhat overestimated; a better estimate might be about -1.4 . In any case, it is quite 
clear that correlation cannot be neglected in this system as it provides one of the most dominant factors in the interaction.

Finally, we compare our calculated interaction energies with experimentally measured quantities. ${ }^{22-24}$ As reported in Table I, the enthalpy of formation of the complex at $298 \mathrm{~K}$ has been measured to lie in the range -4.4 to $-4.6 \mathrm{kcal} /$ mol. Before a direct comparison can be made, the calculated values of electronic energy difference must be combined with zero-point vibrational energies and thermal effects. At 298 $\mathbf{K}$, rotational and translational motions each contribute $-0.9 \mathrm{kcal} / \mathrm{mol}$ to the dimerization reaction; an additional -0.6 arises from the $\Delta P V$ term necessary to convert $\Delta E$ to $\Delta H$. In order to include vibrational considerations, we have adopted the frequencies calculated by Schlegel ${ }^{27}$ using the 4$31 \mathrm{G}$ basis set since there are no available experimental data for the ammonia dimer in the gas phase. With these frequencies, the difference in zero-point vibrational energy is 1.86 $\mathrm{kcal} / \mathrm{mol}$; an additional 2.14 must be added as a result of adjusting the temperature to $298 \mathrm{~K}$. The net result is that a factor of $+1.6 \mathrm{kcal} / \mathrm{mol}$ must be added to the electronic energies in Table I to arrive at an estimate of $\Delta H^{\circ}$ at $298 \mathrm{~K}$. Applying this correction to our MP2/6-31G** $(1 p, 2 d)$ value of -4.05 leads to a theoretical estimate of -2.45 , somewhat smaller in magnitude than experimental measurements. The discrepancy is further increased if BSSE corrections are subtracted from the theoretical data.

Probably the largest source of error in the calculations reported above concerns the approximate nature of the vibrational frequencies. It is well known that frequencies calculated with a basis set of 4-31G type overestimate true values. Moreover, determination of the low-frequency intermolecular vibrations are subject to particularly large errors, an important fact since these low frequencies lead to sizable thermal corrections. We therefore expect that better agreement between theoretical and experimental interaction energies are contingent on an accurate elucidation of the vibrational frequencies in the complex. Other sources of error, which we believe to be of less importance, might be removed by use of larger basis sets and more complete treatments of correlation.

\section{CONCLUSIONS}

Calculated properties of the ammonia dimer are quite sensitive to the size of the basis set and to effects of electron correlation. A basis set with diffuse polarization functions including two sets of $d$-orbitals on nitrogen is necessary for proper treatment of the various components of the interaction energy, for minimization of basis set superposition er- rors, and as an adequate framework for electron correlation. The contributions of correlation to the properties of the complex are substantial, amounting to nearly half of the total interaction energy and reducing the equilibrium internuclear separation by $0.2 \AA$. Indeed, without consideration of correlation, the ammonia dimer is barely bound at all with an enthalpy of dimerization of close to zero.

\section{ACKNOWLEDGMENTS}

We are grateful to Professor Schlegel for permission to use results prior to publication. Financial support was provided by the National Institute of Health (GM29391 and AM01059) and by the Research Corporation. Grants of computer time from SIU are acknowledged.

'G. H. F. Diercksen, W. P. Kraemer, and B. O. Roos, Theor. Chim. Acta 36, 249 (1975).

${ }^{2}$ H. Popkie, H. Kistenmacher, and E. Clementi, J. Chem. Phys. 59, 1325 (1973).

${ }^{3}$ H. Kistenmacher, G. Cilie, H. Popkie, and E. Clementi, J. Chem. Phys. 61, 546 (1974).

${ }^{4} \mathrm{O}$. Matsuoka, E. Clementi, and M. Yoshimine, J. Chem. Phys. 64, 1351 (1976).

${ }^{5}$ B. Jeziorski and M. van Hemert, Mol. Phys. 31, 713 (1976).

${ }^{6}$ M. D. Newton and N. R. Kestner, Chem. Phys. Lett. 94,198 (1983).

'P. A. Kollman and L. C. Allen, J. Am. Chem. Soc. 93, 4991 (1971).

${ }^{8}$ J. D. Dill, L. C. Allen, W. C. Topp, and J. A. Pople, J. Am. Chem. Soc. 97, 7220 (1975).

${ }^{9}$ W. L. Jorgensen and M. Ibrahim, J. Am. Chem. Soc. 102, 3309 (1980).

${ }^{10}$ A. Hinchliffe, D. G. Bounds, M. L. Klein, I. R. McDonald, and R. Righini, J. Chem. Phys. 74, 1211 (1981).

"1J. A. Pople, Faraday Discuss. Chem. Soc. 73, 7 (1982).

${ }^{12}$ K. Ohta, K. Kitaura, Y. Yoshioka, and K. Morokuma, Chem. Phys. Lett. 101, 12 (1983).

${ }^{13} Z$. Latajka and S. Scheiner, Chem. Phys. (submitted).

${ }^{14}$ W. S. Benedict and E. K. Plyler, Can. J. Chem. 35, 1235 (1957).

isJ. S. Binkley, R. A. Whiteside, R. Krishnan, R. Seeger, D. J. DeFrees, H. B. Schlegel, S. Topiol, L. R. Kahn, and J. A. Pople, QCPE, GAussian-80, Program No. 406 (1981).

${ }^{16} \mathrm{C}$. Moller and M. S. Plesset, Phys. Rev. 46, 618 (1934).

${ }^{17}$ J. S. Binkley and J. A. Pople, Int. J. Quantum Chem. 9, 229 (1975).

${ }^{18}$ J. G. C. M. van Duijneveldt-van de Rijdt and F. B. van Duijneveldt, J. Mol. Struct. 89, 185 (1982)

${ }^{19}$ J. W. Reed and P. M. Harris, J. Chem. Phys. 35, 1730 (1961).

${ }^{20} \mathrm{~J}$. Olovsson and D. H. Templeton, Acta Crystallogr. 12, 832 (1959).

${ }^{21}$ A. H. Narten, J. Chem. Phys. 66, 3117 (1977); 49, 1692 (1968).

${ }^{22}$ J. E. Lowder, J. Quant. Spectrosc. Radiat. Transfer 10, 1085 (1970).

${ }^{23}$ J. D. Lambert and E. D. T. Strong, Proc. R. Soc. London Ser. A 200, 566 (1950).

${ }^{24}$ K. D. Cook and J. W. Taylor, Int. J. Mass. Spectrom. Ion Phys. 30, 345 (1979).

${ }^{25}$ E. A. Cohen and A. L. Poynter, J. Mol Spectrosc. 53, 131 (1974).

${ }^{26}$ S. F. Boys and F. Bernardi, Mol. Phys. 19, 553 (1970).

${ }^{27} \mathrm{H}$. B. Schlegel (unpublished results). 